\title{
A Metabolic Activation Mechanism of 7H-Dibenzo[c,g]carbazole via O-Quinone. Part 2: Covalent Adducts of 7H-Dibenzo[c,g]carbazole-3,4-dione with Nucleic Acid Bases and Nucleosides
}

\author{
Weiling Xue, ${ }^{\dagger}$ Angela Siner, ${ }^{\dagger}$ Mark Rance, ${ }^{\ddagger}$ Koka J ayasimhulu, ${ }^{\S}$ \\ Glenn Talaska, ${ }^{\dagger}$ and David Warshawsky*, ${ }^{\dagger}$
}

Department of Environmental Health, Department of Molecular Genetics, Biochemistry, and Microbiology, and Department of Chemistry, University of Cincinnati, Cincinnati, Ohio 45267-0056

Received February 14, 2002

\begin{abstract}
7H-Dibenzo[c,g]carbazole (DBC) is a potent multispecies, multisite carcinogen present in the environment. The metabolic activation pathways of DBC are not completely known. It is hypothesized that DBC may be metabolically activated by oxidation to the reactive Michael acceptor o-quinones, which can form stable and depurinating DNA adducts. The synthesis of DBC-3,4-dione has been previously reported by this research group. In the present article, we describe the synthesis and chemical structural elucidation of nine DBC-nucleic acid adducts produced from reactions of DBC-3,4-dione with Ade, Cyt, 2'-deoxyguanosine (dGuo), 2'deoxycytidine (dCyd), and Guo. Adducts were isolated from reaction mixtures by HPLC and analyzed using MS induding elemental compositions and collision-activated dissociation (CAD), ${ }^{1} \mathrm{H}$ NMR, and two-dimensional chemical shift correlation spectroscopy (COSY) NMR. The adducts, 7-[3,4-dione-DBC-1-yl]-Ade, N44[3,4-dione-DBC-1-yl ]-Cyt, 5-[3,4-dione-DBC-1-yl]-Cyt, two conformational isomers of $\mathrm{N}^{2}-[3,4-d i h y d r o x y-D B C-1-y l]-d G u o$, and two conformational isomers of N2-[3,4-dihydroxy-DBC-1-yl]-Guo, were characterized. Two adducts from reactions of DBC-3,4-dione with dCyd were identified by MS but not fully characterized by NMR due to instability of the adducts. Under similar conditions, the reactions of DBC-3,4-dione with Gua and 2'-deoxyadenosine (dAdo) did not result in an identifiable adduct. Liver DNA adducts from mice treated topically with DBC-3,4-dione (100 $\mu \mathrm{g})$ in dimethyl sulfoxide/acetone $(15 / 85,100$ $\mu \mathrm{L}$ ) were identified with ${ }^{32} \mathrm{P}$-postlabeling. The major adduct chromatographically matched one of the adducts formed from livers of DBC-treated mouse (adduct 3) using identical conditions.
\end{abstract}

\section{Introduction}

As an environmental nitrogen heteropolycyclic aromatic compound (NAH), ${ }^{1}$ 7H-dibenzo[c,g]carbazole (DBC) has been shown to be a potent multispecies, multisite carcinogen in experimental animals with both local and systemic effects $(1,2)$. To elucidate the metabolic activation mechanisms and the ultimate carcinogenic metabol ite(s) of DBC, extensive research has been carried out. Unlike the polycyclic aromatic hydrocarbons (PAHs), DBC metabolism in vitro and in vivo produces mainly monohydroxylated derivatives rather than dihydrodiols (1-5). However, the predominant metabolites produced, 5-hydroxy-DBC and 3-hydroxy-DBC (3-OH-DBC), were not as sarcomagenic by subcutaneous injection as the parent DBC $(1,6,7)$. DBC metabol ism was also expected

\footnotetext{
* To whom correspondence should be addressed.

† Department of Environmental Health.

₹ Department of Molecular Genetics, Biochemistry, and Microbiology.

$\S$ Department of Chemistry.

${ }^{1}$ Abbreviations: DBC, 7H-dibenzo[c,g]carbazole; 3-OH-DBC, 3-hydroxy-DBC; 4-OH-DBC, 4-hydroxy-DBC; PAH, polycyclic aromatic hydrocarbon; $\mathrm{NAH}$, nitrogen heterocyclic aromatic hydrocarbon; HRMS, high-resolution mass spectroscopy; ESI, el ectrospray ionization; CAD, collision-activated dissociation; COSY, two-dimensional chemical shift correlation spectroscopy; DMSO, dimethyl sulfoxide; DMF, dimethylformamide; dGuo, 2'-deoxyguanosine; dCyd, 2'-deoxycytidine; dAdo, 2'-deoxyadenosine.
}

to occur through $\mathrm{N}$-hydroxylation, as is the case of carcinogenic aromatic amines (8,9). After acetylation in situ to trap the unstable monohydroxylated metabolites generated in the DBC incubation mixture with rat liver microsomes, there was no N-hydroxyl-DBC detected (10). Although DBC, which has a relatively low ionization potential (11), can form depurinated DNA adducts through radical-cations by one-electron oxidation (12), this was found to be a minor metabolic pathway in mouse liver and lung (13). An o-quinone was al so tentatively assigned as a minor component in DBC metabolism (3). However, no further examination was carried on at that time due to the lack of information on the role of o-quinone in metabolic activation of PAH and reference standards. Studies in our laboratories have demonstrated that 3-OH- and 4-OH-DBC produced higher levels of DNA adducts in mouse skin, lung, and liver than the parent compound. We thus proposed that 3-OH-DBC is a proximate metabolite $(14,15)$, which needs further activation to an ul timate electrophile and/or redox-active form that is capable of covalently binding with DNA or causing oxidative DNA damage. Referring to the relatively welldocumented metabolic activations of estrogens and homoPAHs $(16,17)$, we hypothesize that 3-OH-/4-OH-DBC may be further bioactivated to yield $\mathrm{DBC}$-o-quinones via DBC-3,4-catechol, as ultimate carcinogenic metabolites 